\title{
A COMPARISON OF THE OKP VISUAL FIELD SCREENING TEST WITH THE HUMPHREY FIELD ANALYSER
}

\author{
STEPHEN A. VERNON ${ }^{1}$ and HARRY A. QUIGLEY ${ }^{2}$ \\ Nottingham, UK and Baltimore, Maryland, USA
}

\begin{abstract}
SUMMARY
In order to determine the loss of retinal sensitivity detectable by oculokinetic perimetry (OKP), we tested 27 eyes of 27 persons with glaucoma and 32 eyes of 32 patients with ocular hypertension with the OKP screener and with a central threshold test on the Humphrey analyser. The threshold at eight locations on the OKP chart between $12.5^{\circ}$ and $15^{\circ}$ from fixation was compared with the corresponding Humphrey perimetric thresholds. Seventeen eyes from 17 patients with glaucoma failed the OKP screening test by not seeing the target at one or more locations. The mean light sensitivity threshold at points seen on OKP screening was $-5.8 \mathrm{~dB}$ (SD $4.6 \mathrm{~dB}$ ) from aged-matched normals (AMN), whereas points missed had a mean threshold -16.1 dB (SD $9.3 \mathrm{~dB}$ ) from AMN. The sensitivity and specificity with which OKP testing differentiated normal points from abnormal ones in Humphrey testing was $82.5 \%$ and $80 \%$ respectively, with a threshold criterion of $-12 \mathrm{~dB}$ from $A M N$. Ten eyes from 10 patients with glaucomatous defects and all of the ocular hypertensive eyes gave normal responses on the OKP screening test. The glaucomatous eyes that passed were characterised by less severe defects on the Humphrey than those who failed in terms of mean deviation $(0.02<\boldsymbol{p}<0.05)$ or corrected pattern standard deviation $(0.01<p<0.02)$. Four of the glaucomatous eyes to pass had a nasal step as the primary field defect. None of those to fail failed only on points within $10^{\circ}$ of fixation. We suggest further modification of the OKP screening chart to improve its efficiency.
\end{abstract}

Oculokinetic perimetry $(\mathrm{OKP})^{\prime}$ uses a wall-mounted screen or hand-held chart to screen for glaucomatous field loss. In the hand-held version (Fig. 1), a central target is surrounded by 26 fixation numbers such that when the patient views a number, the target is located in the visual field within $15^{\circ}$ of fixation. The patient fixates each

'Academic Unit of Ophthalmology, University Hospital, Nottingham, UK.

${ }^{2}$ Dana Center for Preventive Ophthalmology and Glaucoma Service, Wilmer Institute, Johns Hopkins Hospital, Baltimore, Maryland, USA.

Correspondence to: Mr S. A. Vernon, FRCS, FCOphth, Consultant Senior Lecturer, Academic Unit of Ophthalmology, University Hospital, Nottingham, UK. number in turn and reports whether the target is visible at each point on the spiral. This aims to detect scotomas that may be present.

In the UK, only a small percentage of those screening for glaucoma routinely utilise a visual field test. ${ }^{2} \mathrm{OKP}$ therefore has the potential of identifying asymptomatic visual field loss at low cost in individuals at risk of glaucoma.

Initial results with an OKP prototype of the hand-held screener have been published ${ }^{3}$ but, to our knowledge, no report has defined the degree of field defect necessary to produce a positive test. We therefore compared the sensitivity of normal and abnormal response points on the OKP screener to threshold determinations at corresponding points in a group of glaucoma patients with reproducible defects on Humphrey threshold perimetry.

\section{MATERIALS AND METHODS}

Patients attending the Glaucoma Services of the Wilmer Ophthalmological Institute were recruited to the study. Two groups of patients were studied. The first were patients with reproducible defects consistent with glaucoma with the $30 / 2$ or 24/2 full threshold programme of the Humphrey visual field analyser, and optic discs compatible with glaucomatous excavation on biomicroscopy. Only one eye per patient was tested with the OKP screener and eyes were chosen if they displayed relative rather than absolute defects on previous testing with the Humphrey. The second group of patients were ocular hypertensives with normal Humphrey fields in both eyes on at least two occasions. This group had both eyes tested with the OKP screener. All eyes entered into the study had a best corrected acuity of at least $6 / 12(20 / 40)$.

All patients completed the Humphrey test used for the analysis and the OKP test on the same day before formal ophthalmological assessment. Humphrey fields were performed in the usual manner for the programme in question and were followed by the OKP test.

All OKP tests were performed in the same office with diffuse overhead illumination from fluorescent units fitted with a diffuser. Reflected light from the OKP chart was 


\section{GLAUCOMA VISUAL FIELD TEST}

Glaucoma is an eye discase that can cause permanent blindness if not trealed in unie. This screening test
may help you discover an abnormality in your peripheral vision which might be caused by this condition.

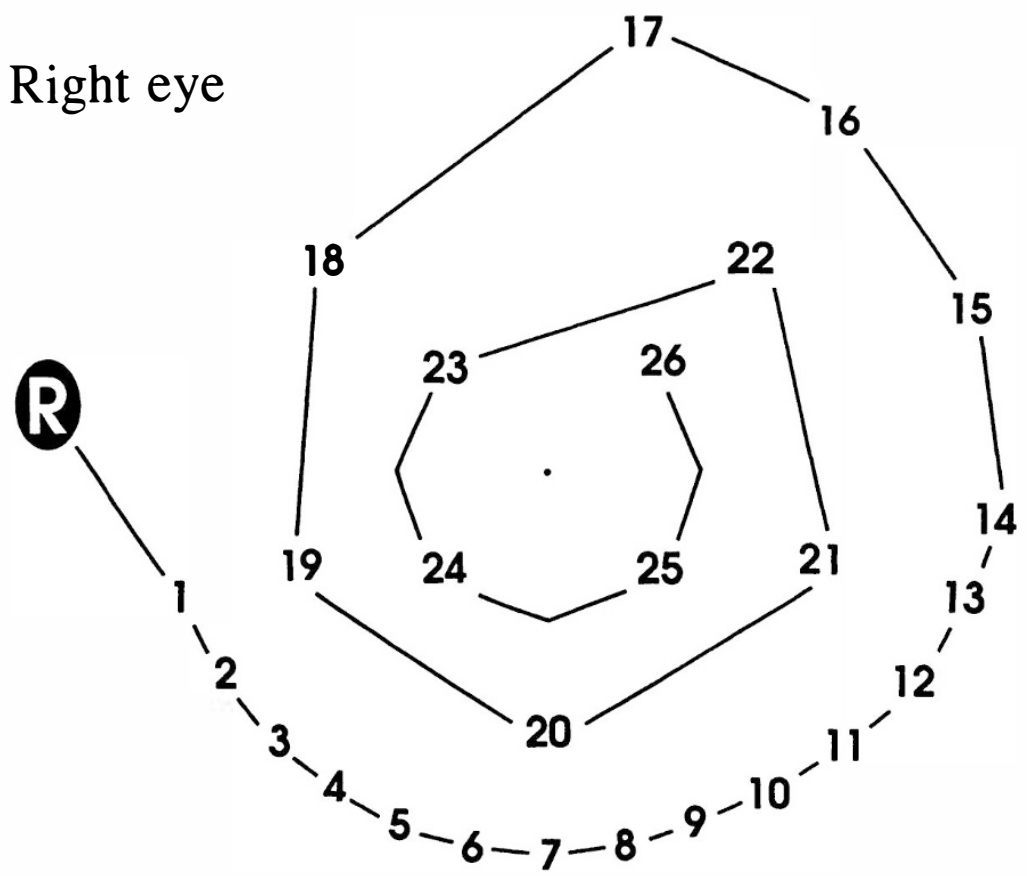

As with any type of test for glaucoma an abnormal result is not necessarly due to glaucomid. or to any other As with any type of test for glaucoma an abnormal result is not necessarly due to glauco

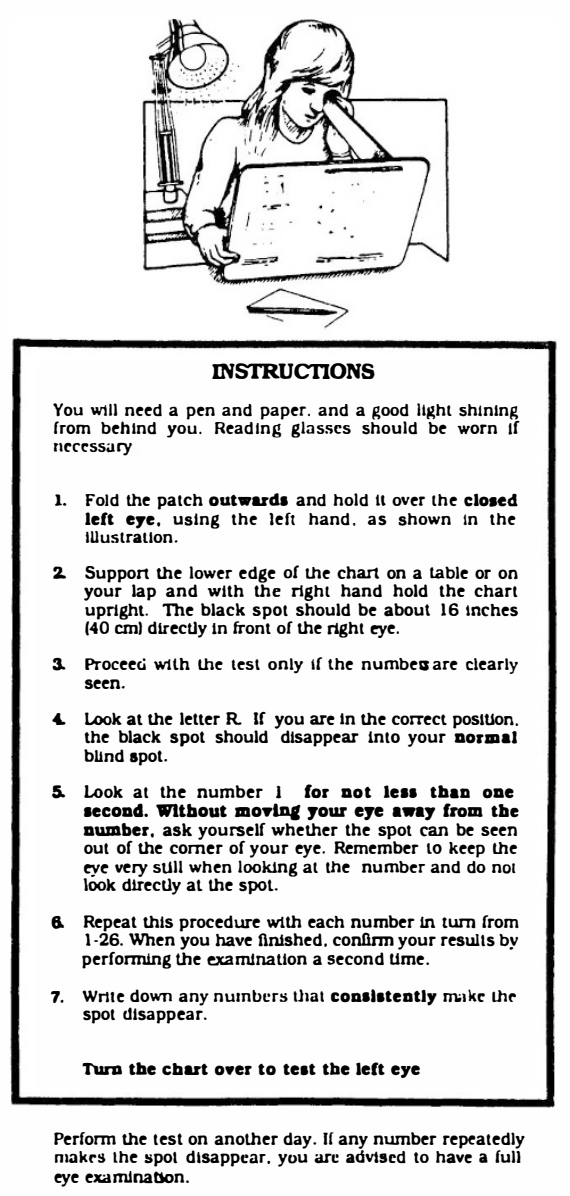

Fig. 1. Hand-held OKP chart designed by Damato using 26 points (right eye).

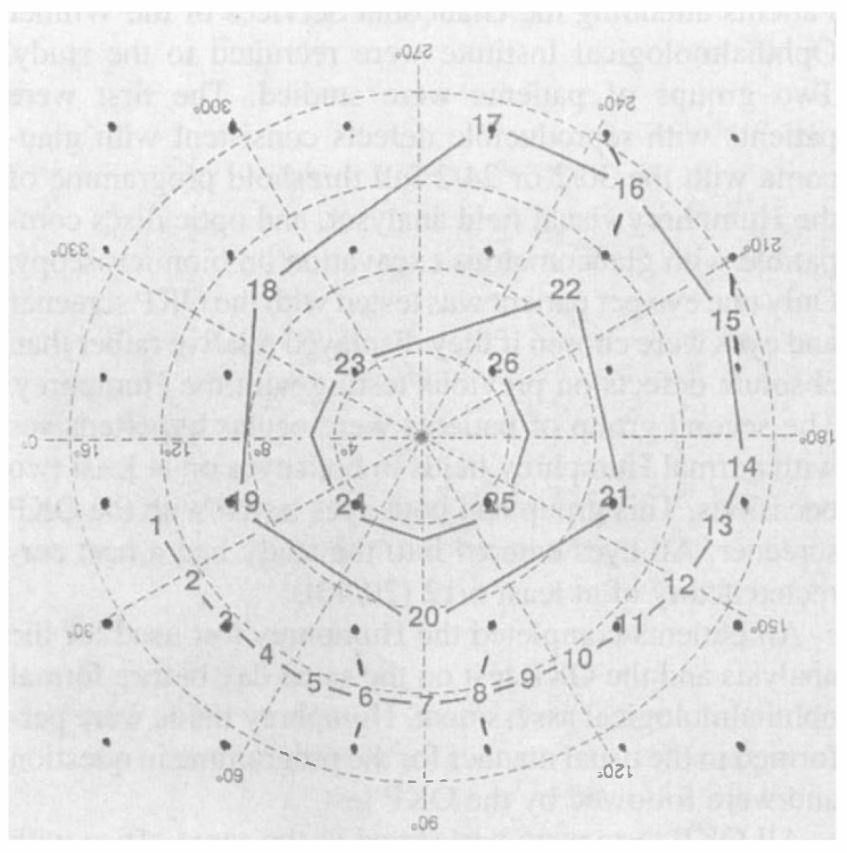

Fig. 2. Position of central Humphrey $24 / 2$ test points superimposed on inverted right eye OKP chart. Thresholds at points $6,8,15$ and 16 were taken as the mean of the two adjacent Humphrey points as shown by dashes. measured at 140 lux ( \pm 10 lux $)$ using a light meter. This was checked several times during the course of the study.

For OKP tests, patients wore a trial frame with $25-\mathrm{mm}$ aperture lenses, with a $2 \frac{1}{2}$ dioptre add over distance correction (the OKP test distance is $40 \mathrm{~cm}$ ). Fellow eyes were occluded. After a brief explanation of the test and the inbuilt blind spot check, patients were asked to follow round the spiral of numbers (Fig. 1). At each number they were to check that the central target dot could be seen in their peripheral field. Patients were asked to spend at least 1 second on each number and to identify any at which the central target could not be seen. Only numbers inducing a 'miss' on two spirals were considered as true defects. A failed test was defined as one or more true defects.

In order to assess the retinal sensitivity at failed points on the OKP test, the positions of the central points on the Humphrey field were superimposed on the OKP interpretation chart (see Fig. 2). Eight points between $12.5^{\circ}$ and $15^{\circ}$ from fixation were identified for analysis. Points $3,11,13$ and 17 were considered to fall on or within $1^{\circ}$ of individual Humphrey threshold points. The threshold equivalents of points $6,8,15$ and 16 were taken as the mean of two adjacent points as identified in Fig. 2.

\section{RESULTS}

Twenty-seven patients with glaucoma whose eyes fulfilled 


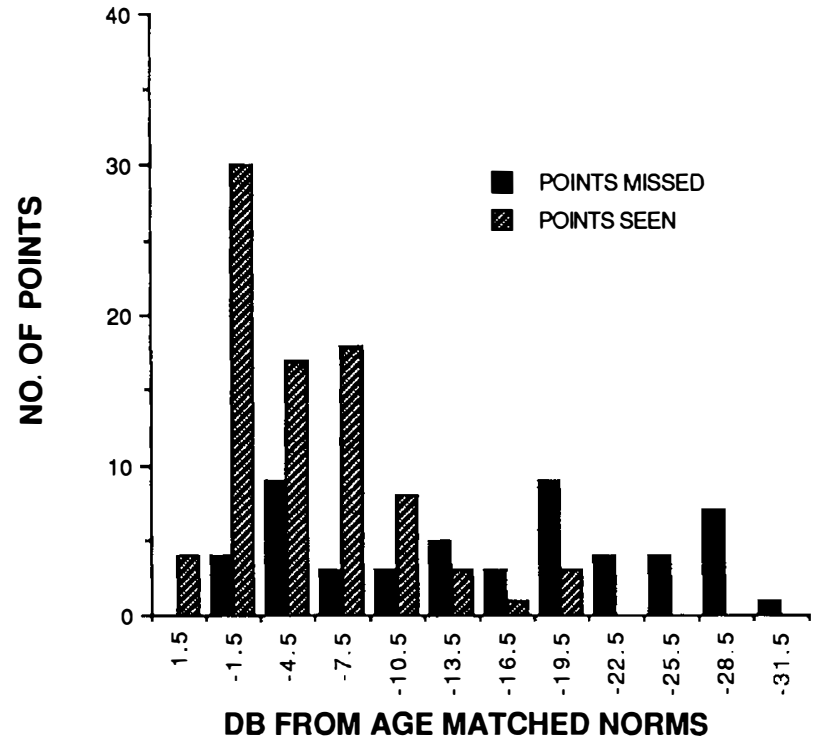

Fig. 3. Threshold distribution of OKP points assessed in the 17 patients who 'failed' the OKP test.

the entry criteria took the OKP test. All eyes with field loss had a score $>3.8$ on the pattern standard deviation (PSD) index and/or $>3.0$ on the corrected pattern standard deviation (CPSD) index resulting in a defect at a statistical level of at least $p<5 \%$ (STATPAC 2 analysis). Seventeen $(63 \%)$ failed the OKP test and 10 ( $37 \%)$ passed. Statistical analysis of inter- and intragroup point thresholds was performed using Fisher's test followed by the Student's $t$ test.

Points missed at the eight positions analysed on the OKP test had a mean threshold $-16.1 \mathrm{~dB}$ (SD $9.3 \mathrm{~dB}$ ) from age-matched normals (AMN). Points seen were at a mean of $-5.8 \mathrm{~dB}(\mathrm{SD} 4.6 \mathrm{~dB})$ from $\mathrm{AMN}(p<0.0001)$. The distribution of thresholds of the 136 points assessed is shown in Fig. 3. The sensitivity/specificity relationship for the OKP screener when detecting a defect at various defect levels identified by the Humphrey is shown in Table I.

A comparison of Humphrey field mean deviation and CPSD between those who passed and failed the OKP test is shown in Table II. Those who passed had fields that were significantly better than those who failed on both measures of field survival. At a mean deviation (MD) level of $<-3$ and $<-6 \mathrm{~dB}$ the sensitivity of OKP was $67 \%$ and

Table I. Sensitivity and specificity of OKP for detecting defects between $12.5^{\circ}$ and $15^{\circ}$ from fixation

\begin{tabular}{lcc}
\hline $\begin{array}{l}\text { Humphrey threshold, } \\
\text { HT }(\mathrm{dB})^{\mathrm{a}}\end{array}$ & Sensitivity $^{\mathrm{b}}$ & Specificity $^{\mathrm{c}}$ \\
\hline-9 & 70.6 & 81.12 \\
-12 & 82.5 & 80.2 \\
-15 & 87.5 & 76.9 \\
-18 & 89.3 & 75.9 \\
-21 & 100.0 & 70.0 \\
\hline
\end{tabular}

${ }^{a}$ From age-matched normals.

b Percentage of OKP points below HT that were missed.

"Percentage of OKP points above HT that were seen.
Table II. Humphrey mean deviation (MD) and corrected pattern standard deviation (CPSD) in glaucomatous eyes that passed and failed OKP

\begin{tabular}{lcrr}
\hline & Failed OKP & Passed OKP & \\
\hline MD (SD) & $-8.3(3.96)$ & $-4.77(3.66)$ & $0.02<P<0.05$ \\
CPSD (SD) & $7.48(2.90)$ & $4.65(2.05)$ & $0.01<P<0.02$ \\
\hline
\end{tabular}

$78 \%$ respectively. At CPSD levels of $>4,>5$ and $>6$ the sensitivity of OKP was $66 \%, 77 \%$ and $92 \%$ respectively.

Thirty-two patients with ocular hypertension took the OKP test, all of whom passed.

\section{DISCUSSION}

Undetected glaucoma accounts for approximately $50 \%$ of cases in population-based studies. ${ }^{4-6}$ In order to detect the maximum number of cases when screening, assessment of the visual field is desirable. The OKP screener provides a simple, rapid, inexpensive method of central field assessment. Its effectiveness is dependent on its sensitivity and specificity and the prevalence of disease in the population in which it is to be used.

The sensitivity and specificity of the test in the community may not equal that calculated in a group of clinic patients who are experienced at field testing and who are being tested under controlled conditions. Test conditions that influence OKP screening include the form of near correction used (small-aperture reading glasses, bifocals with small near segments or varifocals are unsuitable), the background lighting (reduced illumination, reflections and focal irregularities may cause false positives) and poor adherence to the test protocol.

The separation criteria in Table I are calculated from analysis of eyes with glaucomatous field loss. The true specificity of the OKP screener has not been demonstrated to date.

In this study the OKP screener detected $63 \%$ (17/27) of eyes with glaucomatous field loss. However, the eye that was tested was often the less affected eye of a pair. Indeed, in 5 of the 10 eyes that passed OKP screening, there were no points $-10 \mathrm{~dB}$ or more from $\mathrm{AMN}$ in areas tested by OKP.

The efficiency of OKP is further suggested by its sensitivity at detecting eyes with defect levels defined by MD and CPSD. In our subjects, OKP identified $77 \%$ of eyes whose fields had any defective point $>-10 \mathrm{~dB}$ from AMN values. Furthermore, the sensitivity for identifying eyes with CPSD >6 dB was $92 \%$.

It is interesting to note that none of the 17 patients who failed the OKP test on a number $>17$ (i.e. from 17 to 26) on the chart 'passed' all the preceding points. All points numbered $>17$ are within $10^{\circ}$ of fixation where isolated glaucomatous defects may be less common.

Damato and his co-workers attempted to use data from the results of OKP with a 100-number chart on 51 eyes with glaucomatous field loss to position the points on the screener in the most sensitive positions (Fig. 3). ${ }^{7}$ Although these data indicate that points in the inferotemporal field on the chart should be positioned at $12.5^{\circ}$, the 


\section{GLAUCOMA VISUAL FIELD TEST} Glaucoma is an eye disease that can cause permanent blindness if not treated in ume. This screening test
may help you discover an abnomalty in your peripheral vision which might be caused by this condition.

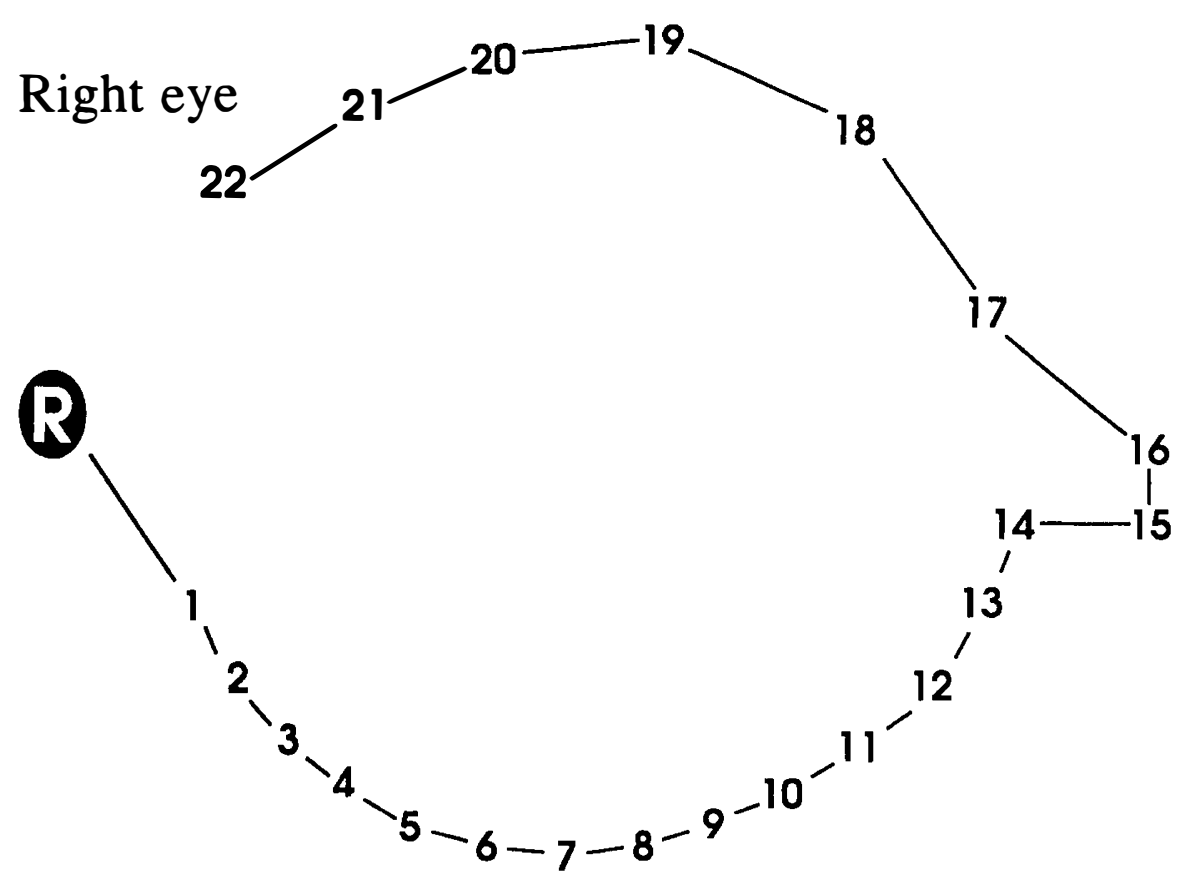

As with any lype of test for glauroma in abnormal regult is not necessartly duc to plaucouma, or in itily wher

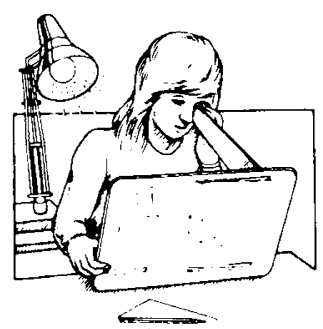

INSTRUCTIONS

You will need a pen and paper. and a good light shining from behind you. Reading glasses should be worn if necessary.

1. Fold the patch outwards and hold it over the closed left eye. using the left hand, as shown in the illustration.

2 Suppon the lower edge of the chart on a table or on your lap and with the right hand hold the char $(40 \mathrm{~cm})$ directly in front of the right eye.

3. Proceed with the test only if the numbesare clearly

4. Look at the letter $R$. If you are in the correct position.
the black spot should disiapprar into your nor mai blund spot.

5. Look at the number 1 for not less than one second. Without moving your eye away from the out of the comer of your eye. Remember to keep the look drectly at the spot.

Repeat this procedure with each number in turn from (1)

7. Write down any numbers that conststeatly make the spot disappear.

Turn the chart over to test the left eye Perform the test on another day. If any number repeatedly
makes the spot disappear. vou are advsed in have it fill eyr eximinition

Fig. 4. Redesigned OKP chart to include nasal step points (15 and 16) and inferotemporal field points 20-22.

points in the inferotemporal field on the OKP chart used in this study are positioned at $10^{\circ}$ from fixation.

We therefore propose that points $18-26$ on the existing chart be replaced by new points $18-22$ as shown in Fig. 4 . This rectifies the error in the positioning of the inferotemporal points and includes two points (15 and 16) designed to detect nasal steps (4 out of the 10 glaucomatous eyes to pass the OKP in our study had a prominent nasal step as the primary field defect).

To assess the sensitivity and specificity of OKP as a screening tool for a community at risk of glaucoma, we must study a representative population where OKP can be tested against a gold standard such as Goldmann or Humphrey perimetry. Before such a large-scale study is commenced, the optimum positioning of the target points should be reconsidered.

Key words: Glaucoma, Humphrey, Oculokinetic perimetry, Screening, Visual field.

\section{REFERENCES}

1. Damato BE: Oculo-kinetic perimetry: a simple visual field test for use in the community. Br J Ophthalmol 1985, 69: 927-31.

2. Vernon SA, Henry DJ: Do optometrists screen for glaucoma? Eye 1989, 6: 743-6.

3. Damato BE, Chyla J, McClure E, Jay JL, Allan D: A hand held OKP chart for screening of glaucoma. Eye 1990, 4: $632-7$.

4. Hollows FC, Graham PA: Intraocular pressure, glaucoma and glaucoma suspects in a defined population. BrJ Ophthalmol 1966, 50: 570-86.

5. Vernon SA, Henry DJ, Cater L, Jones SJ: Screening for glaucoma in the community by non-trained staff using semiautomated equipment. Eye 1990, 4: 89-97.

6. Sommer A, Tielsch JM, Katz J, Quigley HA, Gottsch JD, Javvit J, Singh K: Relationship between intraocular pressure and primary open angle glaucoma in white and black Americans. Arch Ophthalmol 1991, 109: 1090-5.

7. Damato BE, Ahmed J, Allan D, McClure E, Jay JL: The detection of glaucomatous visual field defects by oculokinetic perimeter: which points are the best for screening? Eye 1989, 3: 727-31. 